Linköping Studies in Science and Technology.

Dissertations, No. 1786

\title{
A Comprehensive Analysis of Optimal Link Scheduling for Emptying a Wireless Network
}

\author{
Qing He
}

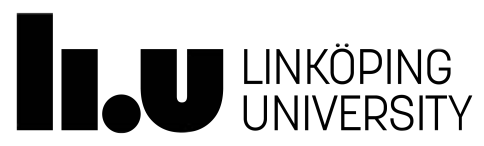

Department of Science and Technology

Linköping University, SE-601 74 Norrköping, Sweden

Norrköping 2016 


\section{A Comprehensive Analysis of Optimal Link Scheduling for Emptying a Wireless Network Qing He}

Linköping Studies in Science and Technology. Dissertations, No. 1786

Copyright (C)2016 Qing He, unless otherwise stated ISBN 978-91-7685-694-9 ISSN 0345-7524 Printed by LiU-Tryck, Linköping, Sweden, 2016 


\section{Abstract}

Wireless communications have become an important part of modern life. The ubiquitous wireless networks and connectivities generate exponentially increasing data traffic. In view of this, wireless network optimization, which aims at utilizing the limited resource, especially spectrum and energy, as efficiently as possible from a network perspective, is essential for performance improvement and sustainable development of wireless communications.

In the dissertation, we focus on a fundamental problem of wireless network optimization, link scheduling, as well as its subproblem, link activation. The problem type arises because of the nature of wireless media and hence it is of relevance to a wide range of networks with multiple access. We freshen these classic problems up by novel extensions incorporating new technologies of interference management or with new performance metrics. We also revisit the problems in their classic setup to gain new theoretical results and insights for problemsolving. Throughout the study, we consider the problems with a general setup, such that the insights presented in this dissertation are not constrained to a specific technology or network type. Since link activation and scheduling are key elements of access coordination in wireless communications, the study opens up new approaches that significantly 
improve network performance, and eventually benefit practical applications.

The dissertation consists of five research papers. The first paper addresses maximum link activation with cooperative transmission and interference cancellation. Papers II and III investigate the minimumtime link scheduling problem in general and a particular class of networks, respectively. In Paper IV, we consider the scheduling problem of emptying a network in its broad form and provide a general optimality condition. In Paper V, we study the scheduling problem with respect to age of information. 


\section{Populärvetenskaplig Sammanfattning}

Trådlös kommunikation är av stor betydelse i dagens samhälle. Möjligheten att ansluta kommunikationsenheter till trådlösa nätverk har ökat markant på senare tid vilket medfört att nätverken belastas alltmer då mängden datatrafik tilltar. Med hänsyn till detta är optimering av trådlösa nätverk synnerligen betydelsefullt. Genom att t.ex. effektivisera nyttjandet av tillgängliga frekvenser samt minska energiförbrukningen är det möjligt att erhålla hållbara kommunikationslösningar med hög prestanda.

Den här avhandlingen fokuserar på optimering i trådlösa nätverk och viktiga utmaningar som berör resurstilldelning och aktivering av förbindelser mellan kommunikationsenheter, vilket kan hanteras på olika sätt och där vald strategi ofta har påtaglig inverkan på ett nätverks prestanda. I avhandlingen studeras klassiska problemställningar på nya sätt med hänsyn till alternativa prestandamått samt tekniker som berör interferens. Det är avgörande att förbindelser och reglering av resurser hanteras på ett vettigt och välgenomtänkt sätt och i avhandlingen ges nya förslag på hur detta kan åstadkommas så att förbättrad nätverksprestanda erhålls, vilket i sin tur skapar bättre förutsättningar för praktiska tillämpningar. De scenarion som beskrivs i avhandlingen är generella vad gäller att slutsatser som presenteras inte är begränsade till en speciell kommunikationsteknik eller nätverkstyp.

Avhandlingen består av fem rapporter. Dessa rapporter beskriver studier där fokus är att maximera antalet aktiva förbindelser, minimera fördröjning och förbättra genomströmning av information samt att reglera nätverksresurser på lämpligt sätt när hänsyn tas till informationens färskhet. 


\section{List of Publications}

\section{Included Papers}

1. Q. He, D. Yuan, and A. Ephremides, "Maximum link activation with cooperative transmission and interference cancellation in wireless networks", IEEE Transactions on Mobile Computing, DOI 10.1109/TMC.2016.2546906.

2. V. Angelakis, A. Ephremides, Q. He, and D. Yuan, "Minimumtime link scheduling for emptying wireless systems: solution characterization and algorithmic framework", IEEE Transactions on Information Theory, vol. 60, no. 2, pp. 1083-1100, 2014.

3. Q. He, V. Angelakis, A. Ephremides, and D. Yuan. "Polynomial complexity minimum-time scheduling in a class of wireless networks", IEEE Transactions on Control of Network Systems, DOI 10.1109/TCNS.2015.2512678.

4. Q. He, D. Yuan, and A. Ephremides, "Optimal link scheduling that minimizes the age of information in wireless systems", IEEE Transactions on Information Theory, submitted; part of the work is published in Proceedings of IEEE International Symposium on Modeling and Optimization in Mobile, Ad Hoc, and Wireless Networks (WiOpt), 2016. 
5. Q. He, D. Yuan, and A. Ephremides, "A general optimality condition of link scheduling for emptying a wireless network", Proceedings of IEEE International Symposium on Information Theory (ISIT), 2016.

\section{Additional Related Publications}

6. E. Karipidis, D. Yuan, Q. He, and E. G. Larsson, "Max-min power control in wireless networks with successive interference cancellation”, IEEE Transactions on Wireless Communications, vol. 14, no. 11, pp. 6269-6282, 2015.

7. Q. He, D. Yuan, and A. Ephremides, "On optimal link scheduling with min-max peak age of information in wireless systems", Proceedings of IEEE International Conference on Communications (ICC), 2016.

8. M. Lei, X. Zhang, T. Zhang, L. Lei, Q. He, and D. Yuan, "Successive interference cancellation for throughput maximization in wireless powered communication networks", Proceedings of IEEE Vehicular Technology Conference (VTC), Fall 2016.

9. Q. He and D. Yuan, "Maximum link activation in wireless networks with cooperative transmission and successive interference cancellation", Proceedings of IEEE International Symposium on Personal, Indoor, and Mobile Radio Communication (PIMRC), 2014. 
10. Q. He, V. Angelakis, A. Ephremides, and D. Yuan, "Revisiting minimum-length scheduling in wireless networks: an algorithmic framework", Proceedings of IEEE International Symposium on Information Theory and its Applications (ISITA), 2012.

11. V. Angelakis, A. Ephremides, Q. He, and D. Yuan, "On emptying a wireless network in minimum time", Proceedings of IEEE International Symposium on Information Theory (ISIT), 2012.

This dissertation is a continuation and an extension of the author's Licentiate thesis.

Q. He, "Revisiting Optimal Link Activation and Minimum-Time Scheduling in Wireless Networks", Licentiate Thesis No. 1695, Linköping Studies in Science and Technology, 2014. 
(iit 


\section{Acknowledgment}

There are so many thanks I would like to say for the countless support and help that I have got during my $\mathrm{PhD}$ studies at the division of Kommunikations- och transportsystem (KTS), Linköping University.

First and foremost, I would like to express my deep and sincere gratitude to my supervisor, Prof. Di Yuan, for his excellent guidance and continuous support during these years. It is my honour to have such an outstanding researcher, who has impressed me by the intelligence, dedication and perfectionism in science, as the mentor of my research work. I have benefited a lot from his selfless knowledge and experience sharing.

I would also like to thank my co-supervisors, Dr. Erik Bergfeldt, Dr. Vangelis Angelakis, and Dr. Scott Fowler, for their kindly support on my studies, teaching, research work, and the writing of this dissertation.

My deep gratitude also goes to Prof. Anthony Ephremides, who is a co-author of the publications included in this dissertation, and from whom I get great support on doing research, writing, and presenting. It is my prized experience to learn from the distinguished professor.

I am grateful to the colleagues in the division of KTS, including all of you who are currently here as well as the former ones who had been 
here since I started my $\mathrm{PhD}$. Thanks for your kindly help, support and the friendly environment you bring to me. Thanks to Lei Lei, Ioannis, who shared not only the office but friendship with me. Thanks to Lei Chen, Sara, for the help on my early-stage studies. Thanks to a long list, Zhuangwei, Joakim, Anders ..., who help me to adapt to the local culture. And also thanks to Viveka, for her always timely support.

Thanks all my friends who help me to live here with a lot of fun! Please forgive me for not finding a way to put all names here.

I appreciate the financial support from the European Union within FP7 Marie Curie funding scheme and Marie Skłodowska-Curie actions in Horizon 2020, the Swedish Research Council (Vetenskapsrådet) and Excellence Center at Linköping - Lund on Information Technology (ELLIIT). Also thanks all who have provided me kindly support during my visits in Ranplan, UK and Forthnet, Greece.

Last but not least, I deeply thank my parents and sister, for the love and support they give me all the time.

Norrköping, August 2016

Qing He 


\section{Abbreviations}

3GPP

3-SAT

4G

5G

AWGN

BFS

BPSK

CDMA

CoMP

CDF

CG

CT

GSM

IC
Third Generation Partnership Project

3-satisfiability

Fourth Generation

Fifth Generation

Additive White Gaussian Noise

Basic Feasible Solution

Binary Phase Shift Keying

Code Division Multiple Access

Coordinated Multi Point Operation

Cumulative Distribution Function

Column Generation

Cooperative Transmission

Global System for Mobile Communications

Interference Cancellation 


\begin{tabular}{ll} 
IoT & Internet of Things \\
ILP & Integer Linear Programming \\
JT & Joint Transmission \\
KKT & Karush-Kuhn-Tucker \\
LA & Link Activation \\
LA-CT-IC & Link Activation with CT and SIC \\
LP & Linear Programming \\
LTE-A & Long Term Evolution Advanced \\
MAC & Media Access Control \\
MCCR & Multi-Cluster Cardinality-based Rates \\
MILP & Mixed Integer Linear Programming \\
MIMO & Multiple Input and Multiple Output \\
MIS & Maximum Independent Set \\
MASP & Minimum Information Age Scheduling Problem \\
MTSP & Minimum Time Scheduling Problem \\
NP & Nondeterministic Polynomial Time \\
OFDMA & Orthogonal Frequency Division Multiple Access \\
RSD & xii \\
\hline MAdio Resource Management
\end{tabular}


SIC

SINR

SNR

STDMA

TDMA
Successive Interference Cancellation

Signal-to-Interference-and-Noise Ratio

Signal-to-Noise Ratio

Spatial Time Division Multiple Access

Time Division Multiple Access 
xiv 


\section{Contents}

I Introduction and Overview 1

1 Introduction . . . . . . . . . . . . . 3

1.1 Motivation ............. 3

1.2 Dissertation Outline and Organization . . . . 4

2 Multiple Access in Wireless Networks . . . . . . . 5

$2.1 \quad$ Basic Scenario . . . . . . . . . . . . 5

2.2 Spatial Time Division Multiple Access . . . 6

3 Link Activation . . . . . . . . . . . . 8

$4 \quad$ Scheduling ................... 11

$4.1 \quad$ Problem Modelling . . . . . . . . . . . . . 12

4.2 Complexity . . . . . . . . . . . 13

$4.3 \quad$ Solutions . . . . . . . . . . . . . . . . 14

$4.4 \quad$ Extensions . . . . . . . . . . . . 15

$5 \quad$ Applied Optimization . . . . . . . . . . . . 16

5.1 Mathematical Modelling . . . . . . . . 16

5.2 Algorithms Involved . . . . . . . . . . . 18

5.3 Complexity Analysis . . . . . . . . . . . 19

6 Contributions . . . . . . . . . . . . . . . . 19 
II Research Papers 41

1 Maximum Link Activation with Cooperative Transmission and Interference Cancellation in Wireless Networks

2 Minimum-Time Link Scheduling for Emptying Wireless Systems: Solution Characterization and Algorithmic Framework

3 Polynomial Complexity Minimum-Time Scheduling in a Class of Wireless Networks

4 A General Optimality Condition of Link Scheduling for Emptying a Wireless Network

5 Optimal Link Scheduling That Minimizes the Age of Information in Wireless Systems 


\section{Part I}

\section{Introduction and Overview}





\section{Introduction}

\subsection{Motivation}

Wireless communications have been developing rapidly since the 1980s. Various wireless systems have been deployed globally to support ubiquitous connectivities between people and people, people and device, and device and device. Notable amongst them are mobile telecommunications and Internet of Things (IoT). The former enables communications between people at anytime and anywhere, while the latter intends to have everything connected.

The ever increasing number of users and constantly emerging applications generate more and more data traffic. The prediction by Cisco shows that the global mobile data traffic will increase nearly eightfold between 2015 and 2020 [1]. However, the resource of wireless networks, especially spectrum and energy, are limited [2, 3]. To support the explosively increasing data traffic, new physical-layer transmission technologies, such as orthogonal frequency division multiple access (OFDMA), multiple-input and multiple-output (MIMO) [4, 5], have been deployed $[6,7]$. Another line towards capacity improvement and sustainable development of wireless communications is network optimization, which aims to utilize the resource as efficiently as possible from a network perspective. Wireless network optimization covers a variety of topics such as radio resource management, routing, crosslayer optimization, and so on.

Nowadays a variety of physical-layer technologies and network topologies are used in wireless systems. For example, the physical-layer specifications of mobile telecommunications evolve by each genera- 
tion; cellular networks are infrastructure-based while sensor networks usually follow an ad hoc topology. In the vision of $5 \mathrm{G}$ and beyond [8], various network types are expected to coexist and may merge to-

gether resulting in new types like heterogeneous networks. In view of that, wireless network optimization based on specific problem settings or network configurations has its limitation. The derived results are restricted to the networks in question and hence have to be revisited when being extended to other systems. Moreover, by incorporating new emerged technologies of interference management, some classic optimization problems call for further investigation. In this dissertation, we focus on one of the fundamental problems in wireless network optimization, link scheduling, as well as link activation, which is a core building block of the scheduling problem. We study the problems with a general setup or novel extensions, to gain insights that are of importance to a wide range of wireless networks.

\subsection{Dissertation Outline and Organization}

The dissertation consists of two parts. In Part I, we provide a brief introduction including background knowledge of wireless network optimization, as well as an overview of the author's work. In Part II, five research papers are presented.

Part I is organized as follows. In Section 2, multiple access in wireless networks is introduced, followed by classic schemes of access coordination. Sections 3 and 4 are dedicated to link activation and scheduling, respectively. Mathematical background of applied optimization is given in Section 5. In Section 6, we summarize the research papers in Part II and outline the contributions. 


\section{Multiple Access in Wireless Networks}

\subsection{Basic Scenario}

Due to the broadcast nature of wireless media, the transmitter-receiver pairs, or links, that share a common channel cause interference to each other if they are active simultaneously. Therefore a key aspect of access coordination in a wireless network with multiple access is to decide when and which of these mutually interfering links should transmit and for how long. We illustrate in Figure 1 a basic scenario where $N$ links sharing a common channel.

Note that, this scenario represents a general case that exists in various networks such as mobile networks, sensor networks, and so on. Hence, throughout this dissertation, we start the study of our optimization problems from this basic scenario, and whenever applicable, extend it to more complicated ones.

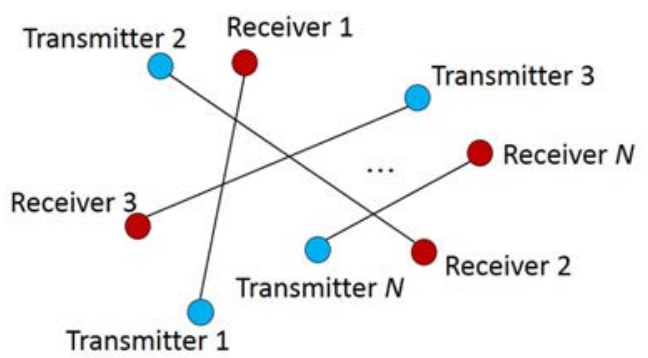

Figure 1: A basic scenario. 


\subsection{Spatial Time Division Multiple Access}

For a set of co-channel links, a simple solution of access control is time division multiple access (TDMA). In this scheme, links are activated separately in their respective time slots. In each time slot, only one link is allowed to transmit and hence suffers no interference from others. TDMA is easy to implement and has been used in many wireless systems, e.g., global system for mobile communications (GSM), where links sharing the same channel of a cell transmit in different time slots.

However, TDMA may result in a poor capacity utilization due to the exclusivity in link transmission. In [9], the authors proposed spatial time division multiple access (STDMA), which is an extension of TDMA. The principle of STDMA is to simultaneously activate links that are collision-free in every time slot. To quantitatively estimate the interference and determine whether a link collides with others or not, two interference models have been used, as illustrated in Figure 2.

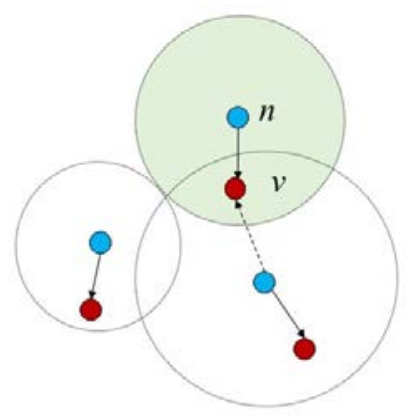

Protocol Model

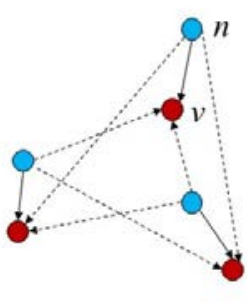

Physical Model

Figure 2: Interference models.

The protocol model had been commonly used by the networking 
community in early time. We use "node" to denote the transmitter and reviver in the graph. For a set of nodes $\mathcal{V}$ on an Euclidean plane, each node $n \in \mathcal{V}$ is associated with a disk of radius $r(n)$, which depends on the transmission power level $P_{n}$ and the channel condition. The signal from node $n$ is assumed to be "heard" only within this range (as shown by the light green circle in Figure 2). This results in a graph $\mathcal{G}=(\mathcal{V}, \mathcal{E})$, where set $\mathcal{E}$ is obtained by adding edges between $n$ and $v$, $\forall n, v \in \mathcal{V}$, if $d(n, v) \leq r(n)$, where $d(n, v)$ denotes the Euclidean distance between nodes $n$ and $v$. Interference in this, so called, "conflict graph" is modelled through the constraint: if node $n$ transmits to $v$, no adjacent node of $v$ should transmit concurrently.

The physical model is considered in $[10,11]$, utilizing the signalto-interference-and-noise ratio (SINR) to measure channel quality. It is based on practical transceiver designs of communication systems that treat interference as noise. The SINR that node $v$ experiences is quantified by the following formula (1).

$$
\mathrm{SINR}_{v} \triangleq \frac{G_{n v} P_{n}}{\sum_{m \neq n} G_{m v} P_{m}+\sigma_{v}^{2}},
$$

where $\sigma_{v}^{2}$ is the noise power at receiver node $v$ and $P_{n}$ is the transmission power of transmitter $n$. Parameter $G_{m v}$ is the propagation gain between nodes $m$ and $v$, incorporating the effects of path loss, shadowing, and fading.

Under the physical model, which is also referred to as the SINR model, a transmission is successful means that the SINR value at the intended receiver exceeds a threshold so that the transmitted signal can be decoded with an acceptable bit error probability $[12,13]$. The physical model takes into consideration the effects of interference and power 
on the transmission rate (corresponding to the SINR threshold), and hence is more realistic on modelling the wireless channel $[14,15]$.

\section{Link Activation}

Link activation (LA) is one of the fundamental problems of radio resource management (RRM). It aims to answer the question which links can be simultaneously active in a shared channel. Under the protocol model, LA amounts to identifying independent sets in the conflict graph of the network. Following the physical model, the SINR constraint must be satisfied for each active link. The selection of link set, or group, is driven by some optimization criterion. A basic version of LA, a.k.a., maximum LA, is to maximize the cardinality of the set of links that are compatible, i.e., can concurrently transmit. Maximum LA is a special case of maximum weighted LA, in which each link is associated with a positive weight and the objective is to maximize the total weight of activated links. The weights may represent utility, queue size, or some virtual metrics such as linear programming (LP) dual prices, which are used for the column generation method for the scheduling problem to be discussed in Section 4.

LA is an optimization problem of combinatorial nature. The maximum weighted LA problem accepts an integer programming (IP) model with the SINR constraint for each activated link under the physical model, or the constraints that all the activated links are disjoint in the conflict graph under the protocol model.

The LA problem has been studied extensively in the past [16]. Theoretical results and algorithms for LA under the protocol model are 
discussed in [17]-[23], etc. LA under the physical model is proved to be nondeterministic polynomial time $(N P)$-hard in $[12,24]$. The hardness of the LA with geometric gain matrix is established in [25], where a heuristic algorithm is included. Approximation algorithms for LA under the physical model are provided in $[12,26]$. Algorithms with constant approximation guarantee are discussed in [24, 27], under the uniform power assumption. In [28], a constant-factor approximation algorithm for the general case of variable power is developed. In [29], the authors proposed a new approach for the global optimal solution of the LA problem, with an effective representation of the SINR constraints to avoid numerical instability.

LA is a key element in scheduling. A feasible schedule with the STMDA scheme is composed by link subsets, each of which is a solution of LA. In that sense, LA is also referred to as "one-shot scheduling" or "one-slot scheduling" [25]. Research on scheduling, which uses LA as the building block, is extensive, e.g., [30, 31, 32]. LA is also an integral part of more complicated, cross-layer optimization problems that jointly consider scheduling and other resource control aspects, such as rate adaptation and power control, as well as routing, in ad hoc and mesh networks (see [33, 34]).

The LA problem can be extended by incorporating the following new technologies of interference management and power control.

- Cooperative transmission (CT)

The idea of cooperation at the transmission layer originates from using multiple relays whose transmissions are combined at the receiver [35, 36]. In [37, 38], protocols and performance gain for cooperative diversity are discussed. Capacity analysis for cooper- 
ative relaying has been presented in $[39,40]$. Recently, CT, a.k.a., joint transmission, is regarded as one of the primary techniques of coordinated multi point (CoMP) operation, which has been introduced as a promising way to improve network performance at cell edges for LTE-Advanced [43, 44].

- Interference cancellation (IC)

IC is one of the key features in Long Term Evolution Advanced (LTE-A) heterogeneous networks [6, 45]. To perform IC, receivers should have multiuser detection capability and sufficient information, such as coding schemes and type of modulation, to decode interfering signals, whenever these interfering signals are strong enough [46]. If the interfering signals are cancelled one by one, that is, they are decoded and then subsequently subtracted from the total sum of interference, we call it successive IC (SIC). Theoretically, a receiver can cancel up to $N-1$ interfering signals in a network with $N$ links in total. However, due to practical issues, e.g., hardware capacity, the number of cancellation stages may have to be limited.

In contrast to the conventional approaches that try to decrease the strength of interfering signals, with IC, it might be favourable to strengthen interference in order to enable IC and ultimately increase the achievable SINR. The improvement of network capacity by IC are studied in [47, 48]. For LA, in [49], the authors addressed optimal LA under the physical model with IC, indicating the effectiveness of IC in boosting the number of concurrently activated links, especially for low to medium SINR thresholds. 
It is worth noting that $\mathrm{CT}$ and IC are complementary, as they enhance and decrease the numerator and denominator of the SINR, respectively. In Paper I, we study a novel extension of LA by joint considering the two new features. Numerical study shows that CT and SIC result in a synergy that significantly improves the number of concurrently active links.

- LA and power control

Power control is also a classic topic in RRM. It aims to determine transmission power for each active link so that an optimality goal is achieved. By employing optimized power assignment, we can either improve the solution of LA or increase the achievable SINR of all active links. Moreover, it is possible to further extend the optimization problem by incorporating SIC. In [50] (the 6th paper in the publication list), max-min power control for a set of active links with SIC enabled is studied. The results demonstrate remarkable gains in the common achievable SINR, especially for high-interference scenarios.

\section{Scheduling}

Link scheduling in a wireless network is to organize the transmission of links sharing a common channel. It consists of the fundamental questions of when and which of the mutually interfering links should transmit so that some criteria, such as throughput, energy, time, or their combinations, is optimized. There is a rich amount of literature available for the scheduling problem (see the surveys [16, 31, 32, 51, 52] and the references therein). The study of the scheduling problem has 
ranged from purely network-level approaches with the protocol model to fully cross-layered setups that integrate into the overall network resource allocations.

\subsection{Problem Modelling}

The modelling of the scheduling problem varies by the network assumptions. For scheduling with the STDMA scheme, a common constraint is that each active link set, or group, is compatible, i.e., it is a feasible solution of LA, which has been discussed in Section 3. In [53], the authors provide a formulation of the scheduling problem based on the assumption that the traffic is bursty and the objective is to maximize the stable throughput region of the network, where a network is stable if none of the queues grows without bound. This formulation has led to a fairly general solution that is known as the "back-pressure" algorithm, which provides broad insights of the scheduling problem and leads to many extensions (e.g., [52, 54, 55]). An alternative way of modelling the scheduling problem follows the way of emptying a network, where each link is associated with a finite amount of demand, which should be emptied by the end of a schedule. With the objective of emptying the demand in minimum time, we have the so called minimum-length scheduling, or, minimum-time scheduling (MTSP) [56]-[60]. Under the setup of emptying a network, minimizing transmission time is also equivalent to a form of throughput maximization. This modelling view applies to the networks operating at both stationary and ergodic environments or not, and holds promise towards evaluating the ultimate capabilities of networks [32]. In this dissertation, we study the scheduling problem of emptying a network in Papers II-V. 
For scheduling with LA as the building block, the problem can be formulated as an IP model with the objective of optimizing a given cost criterion. A summary of formulations is presented in [16, 31]. If the time slot can be arbitrarily small, i.e., the activation duration of a compatible link set can be any non-negative value, the scheduling problem accepts an LP model (with exponential size in general). From an information theoretical perspective, the capacity regions for some transmission strategies with a given schedule are studied in [61]. In Paper V, we combine the methodologies used in information theory and networking performance engineering, to achieve a general optimality condition of the scheduling problem.

The scheduling problem has been considered with various performance metrics. For scheduling with minimum energy, it is proved that TDMA is optimal if the transmission time is not of concern [62]. Suppose now the metric is the end-to-end delay. For this metric, the optimal schedule coincides with MTSP for single-hop networks, no matter the given demand is periodically repeated or not; but for a multi-hop network, the results differ for these two cases [63]. In Paper IV, we consider the scheduling problem with a new metric, age of information, which measures the freshness of information and of which the importance has been recently recognized.

\subsection{Complexity}

For the problem complexity, the general hardness of the scheduling problem under the protocol model is provided in [64]-[67]. Under the physical model, the problem with an arbitrary gain matrix, is proved to be $N P$-hard in [68], by a polynomial-time reduction from the graph 
coloring problem. In [25], $N P$-hardness of the scheduling problem with a geometric gain matrix is addressed. Although the problem is hard in general, some special cases allow for a polynomial algorithm $[56,57]$. In Paper III, we identify a class of tractable cases where the link rates have a particular structure.

\subsection{Solutions}

A variety of algorithm design and problem approximations have been proposed and studied for the scheduling problem, e.g., [17, 31], [69][72]. Under the protocol model, graph-based scheduling algorithms employing implicit or explicit coloring strategies are widely used, e.g., $[73,74]$. In [75, 76], greedy-type heuristic algorithms are developed for the MTSP. Optimal and approximation algorithms for scheduling in networks of trees and planar graphs are provided in [77]. The problem of scheduling broadcast messages is studied in [78, 79, 80] and distributed algorithms thereof are discussed. In [81], a neural network approach is proposed.

For the scheduling problem with SINR constraints, i.e., under the physical model, many heuristic algorithms and problem approximations have been investigated, e.g., [26, 27], [82]-[85]. In [86], the MTSP is formulated as a shortest path problem and sub-optimal analytic characterizations are obtained. In [30,68], the authors showed that scheduling in wireless networks can be represented by a set-covering formulation of IP and thus accepts a column generation method for solving the resulting LP relaxation. In [87], a column generation based solution method is applied to approach an optimal solution, with a potential advantage of not having to enumerate all compatible link sets 
a priori. In [88], the authors compared two methods for identifying the compatible set in the pricing problem of column generation. For the scheduling problem with geometric gain matrix, a heuristic algorithm is investigated in [25]. In Paper II of this dissertation, we provide a modular algorithmic framework to encompass exact as well as suboptimal, but fast, scheduling algorithms, all under a unified design principle.

\subsection{Extensions}

The joint problem of scheduling and power control in ad hoc networks is addressed in [89]. The optimization of scheduling with power control and rate adaptation in wireless mesh networks is studied in [90]. Joint rate control and scheduling in multihop wireless networks is presented in [91]. The authors of [92] discussed the problem with the objective of minimizing the total transmit power subject to end-to-end bandwidth guarantees and bit error rate constraints of each communication session. The scheduling problem of maximizing data throughput by adaptive modulation and power control is investigated in [93]. In [59], the authors included the aspects of the routing problem and utilized column generation to compute the solution. The joint problem of routing and scheduling is also studied in [42] and [94], for ad hoc networks and wireless mesh networks with directional antennas, respectively. A comprehensive survey of extensions of the scheduling problem with other resources allocation policies is presented in [16].

The scheduling problem with IC is considered and proved to be $N P$-hard in [95] and an IP model is formulated therein. In [96], a greedy algorithm for scheduling with SIC is evaluated. In [97], the 
authors considered joint back-pressure power control with single-stage IC. Rate selection with IC to maximize throughput is discussed in [98].

\section{Applied Optimization}

To solve network optimization problems, mathematical modelling and applied optimization are commonly used [99, 100]. In this section, we provide background knowledge of the optimization methods used in the dissertation.

\subsection{Mathematical Modelling}

- Linear programming (LP)

LP refers to a problem of optimizing a linear function over a region specified by linear constraints. The standard form of an LP is written as:

$$
\begin{array}{ll}
\operatorname{minimize} & \mathbf{c}^{\mathbf{T}} \mathbf{x} \\
\text { subject to } & \mathbf{A x}=\mathbf{b} \\
& \mathbf{x} \geq \mathbf{0}
\end{array}
$$

Here $\mathbf{c}=\left(c_{1}, c_{2}, \ldots, c_{n}\right)^{T}$ is a given cost vector, and $\mathbf{x}$ is an $n$ dimensional decision vector. A vector $\mathrm{x}$ satisfying all constraints is a feasible solution. A feasible solution that minimizes the objective is an optimal solution, and the corresponding objective value is called the optimal cost. For more information of LP, the readers can refer to [101].

- ILP and MILP 
If all variables of an LP are restricted to be integer, then the optimization problem is an integer linear programming (IP or ILP); if only part of the variables are integers, then the problem is a mixed integer linear programming (MIP or MILP). More information about ILP and MILP can be found in [102].

For ILP and MILP, problem instances of up to moderate size can be solved by the algorithms, e.g., branch-and-bound, branch-andcut, using off-the-shelf optimization solvers like CPLEX [104] and Gurobi [105].

- Convex optimization

A convex optimization problem is an optimization problem that minimizes a convex objective function or maximizes a concave objective function over a convex set. A convex optimization problem can be written as:

$$
\begin{array}{cl}
\operatorname{minimize} & f_{0}(x) \\
\text { subject to } & f_{i}(x) \leq 0, i=1, \ldots, m \\
& a_{i}^{T} x=b_{i}, i=1, \ldots, n,
\end{array}
$$

where $f_{0}, \ldots, f_{m}$ are convex functions and the equality constraint functions in (3c) are affine.

Convex optimization is a powerful method on problem solving, since it allows for the computation of a global optimal solution by solvers and there are theoretical results available in convex analysis [103]. 


\subsection{Algorithms Involved}

- Column generation (CG)

Column generation (CG) is an extension of the simplex algorithm, which is used to solve LP. The algorithm CG starts from a master problem, which is a restricted version of the original optimization problem, then solves the pricing problem aiming to identify the column with the most negative reduced cost. That column is then added to the master problem. The algorithm repeatedly solves the master problem and the pricing problem, until no column with negative reduced cost can be found by the pricing problem. The algorithm CG is usually applied to solve large-sized LP in which the number of variables is much more than that of constraints, with the advantage of a potentially reduced complexity.

- Heuristic algorithm

A heuristic algorithm is designed to solve a problem in a fast and efficient way without global optimum guarantee. An example is greedy algorithm, in which the locally optimal choice is taken at each step, with the hope of finding a good, or even optimal, solution. Local search is also a heuristic method. The algorithm moves from its current solution to its neighbours, which are defined by modifying part of the current solution, until no further improvement can be made.

More details of algorithms in applied optimization can be referred to $[106,107]$. 


\subsection{Complexity Analysis}

Both LA and scheduling fall into the domain of combinatorial optimization, for which complexity is a fundamental aspect. If the problem is proved to be $N P$-hard, then one cannot expect an algorithm that solves the problem in polynomial time (in the size of problem input) with global optimum guaranteed, unless $P=N P$.

The proof of $N P$-hardness is usually achieved by showing that the problem in question is a polynomial reduction from a known hard problem. In this dissertation, we have utilized the following problems as well as their variations in problem reduction: partition, maximum independent set (MIS), coloring, and 3-satisfiability (3-SAT). Detailed treatment of complexity is available in [108].

\section{Contributions}

We outline the scientific contributions of the dissertation along three lines.

- Addressing "new problems"

We renew the classic problems by: (i) investigating new extensions, specifically, in Paper I, we study LA with a novel setup incorporating CT and SIC; (ii) considering new performance metrics, specifically, in Paper V, we propose to optimizing link scheduling with respect to age of information, which is a new metric that measures the freshness of information.

- Revisiting the classic problem and providing new insights 
In Papers II and III, we revisit the MTSP, deriving fundamental results in complexity, optimality conditions, and tractable cases, as well as designing algorithms and problem solutions.

- Deriving a unified characterization of optimal scheduling We study the scheduling problem in its general form in Paper IV, and provide fundamental insights that lead to a unified treatment for characterizing the optimal scheduling.

The dissertation consists of five papers, for which the main ideas, key theoretical results, major concepts of solution approaches, and most of numerical studies, are results of the discussion among all authors. The author of the dissertation is the first author of Papers I, III, IV, and V, taking the responsibility of all contents of the papers, including writing. For Paper II, the author has mainly contributed to the theoretical results of the cardinality-based rates, optimality property of the algorithms, as well as simulation study. The research work of the papers is summarized as follows.

\section{- Paper I: Maximum Link Activation with Cooperative Trans-} mission and Interference Cancellation in Wireless Networks

We address the maximum link activation problem in wireless networks with new features, namely when the transmitters can perform cooperative transmission, and the receivers are able to perform successive interference cancellation. In this new problem setting, which transmitters should transmit and to whom, as well as the optimal cancellation patterns at the receivers, are strongly intertwined. 
In this paper, first we provide a thorough tractability analysis, proving the NP-hardness as well as identifying tractable cases. Second, for benchmarking purposes, we deploy integer linear programming for achieving global optimum using off-the-shelf optimization methods. Third, to overcome the scalability issue of integer programming, we design a sub-optimal but efficient optimization algorithm for the problem in its general form, by embedding maximum-weighted bipartite matching into local search. Simulation results demonstrate that cooperative transmission and successive interference cancellation have a clear complementary effect in yielding significant gain on improving the number of links that can be activated concurrently. Moreover, the proposed algorithm is effective in exploiting the joint effect of the two schemes. The paper is published in IEEE Transactions on Mobile Computing, 2016.

\section{- Paper II: Minimum-Time Link Scheduling for Emptying Wire-} less Systems: Solution Characterization and Algorithmic Framework

We consider the minimum-time scheduling problem for the case of emptying $N$ queues over a shared channel, with generic consideration of rates, which may be produced by some underlying function, unifying the previously considered formulations.

In this paper, we present fundamental insights and solution characterizations that include: (i) showing that the complexity of the problem remains high for any continuous and increasing rate func- 
tion, (ii) formulating and proving sufficient and necessary optimality conditions of two base scheduling solutions that correspond to emptying the queues using "one-at-a-time" or "all-atonce" strategies, (iii) presenting and proving the tractability of the special case in which the transmission rates are functions only of the cardinality of the link activation sets. These results are independent of physical-layer system specifications and are valid for any form of the rate function. We further develop an algorithmic framework that encompasses exact as well as sub-optimal, but fast, scheduling algorithms, all under a unified principle design. Through computational experiments we finally investigate the performance of a host of specific algorithms from this framework.

The paper is published in IEEE Transactions on Information Theory, 2014.

\section{- Paper III: Polynomial Complexity Minimum-Time Schedul-} ing in a Class of Wireless Networks

We consider the minimum-time scheduling problem, which has been proved to be NP-hard in general, and identify tractable cases.

In this paper, we prove that this problem remains hard even for the case in which the receivers are co-located. Then we study a class of minimum-time scheduling problems in which the link rates have a particular structure. Specifically, we consider the case with multi-cluster cardinality-based rates, showing that it can be 
solved in polynomial time and presenting conditions for problem decomposition. The assumed model is extended to more general wireless networks with $k$-means clustering, and a column generation algorithm has been designed. We apply the proposed approach to exactly solve the problem or provide good approximations, as well as lower and upper bounds for the optimal solution. Numerical results are provided to evaluate the performance of the approach. It is demonstrated that, with a moderate number of clusters in the approximation, the optimality gap of no more than 4.0 percent on average can be reached.

The paper is published in IEEE Transactions on Control of Network Systems, 2015.

\section{- Paper IV: A General Optimality Condition of Link Schedul-} ing for Emptying a Wireless Network

We consider link scheduling in wireless networks for emptying the queues of the source nodes in its general form, and investigate optimal scheduling not only in the sense of deciding which nodes transmit and for how long, but also at what bit-rate for given power levels and channel characteristics.

In this paper, we provide a unified mathematical formulation of the scheduling problem that accommodates all meaningful settings of link transmission rates and network configurations, treated or untreated in the literature. A series of theorems are proved to establish that any scheduling problem is equivalent to solving a convex problem defined over the convex hull of the rate region. 
Based on the fundamental insight, a general optimality condition is derived, that yields a unified treatment of optimal scheduling. Furthermore, we demonstrate the implications and usefulness of the result. Specifically, by applying the theoretical insight to optimality characterization and complexity analysis of scheduling problems, we can both unify and extend previously obtained results.

The paper is published in Proceedings of IEEE International Symposium on Information Theory (ISIT), 2016, and is under preparation of submitting to IEEE Transactions on Information Theory.

\section{- Paper V: Optimal Link Scheduling That Minimizes the Age of Information in Wireless Systems}

There is a growing interest in the concept of the age of information, which is a recently recognized metric that describes the freshness of information in communication systems. We investigate the age of information in a wireless network and propose a novel approach of optimizing the scheduling strategy to deliver all messages as fresh as possible. Specifically, we consider a set of links that share a common channel. The transmitter at each link contains a given number of packets with time stamps from an information source that generated them.

In this paper, we consider wireless link scheduling under the concept of age of information and address the minimum age scheduling problem (MASP). The MASP is different from minimizing the time or the delay for delivering the packets in question. We model the problem mathematically and prove it is $N P$-hard in 
general. We also identify tractable cases as well as optimality conditions. An integer linear programming formulation is provided for performance benchmarking. Moreover, a steepest age decent algorithm with better scalability is developed. Numerical study shows that, by employing the optimal schedule, the overall information age is significantly reduced in comparison to other scheduling strategies.

The paper is submitted to IEEE Transactions on Information Theory. Part of the work is published in Proceedings of IEEE International Symposium on Modeling and Optimization in Mobile, Ad Hoc, and Wireless Networks (WiOpt), 2016. 
6. CONTRIBUTIONS

26 


\section{Bibliography}

[1] Cisco. Visual networking index (VNI) global mobile data traffic forecast. White Paper, 2015. Available at http://www.cisco.com.

[2] ITU. Radio Regulations, 2012. Available at http://www.itu.int.

[3] A. Fehske, G. Fettweis, J. Malmodin, and G. Biczok. The global footprint of mobile communications: the ecological and economic perspective IEEE Commun. Mag., vol. 49, no. 8, pp. 55-62, Aug. 2011.

[4] D. Tse and P. Viswanath. Fundamentals of Wireless Communications. Cambridge University Press, 2005.

[5] E. G. Larsson and P. Stoica. Space-Time Block Coding for Wireless Communications. Cambridge University Press, 2003.

[6] 3GPP. Available at http://www.3gpp.org/.

[7] 3GPP2. Available at http://www.3gpp2.org/.

[8] EU 5G-PPP. The next generation of communication networks and services. $5 G$ Vision, 2015. Available at https://5g-ppp.eu. 
[9] R. Nelson and L. Kleinrock. Spatial TDMA: a collision-free multihop channel access protocol. IEEE Trans. Commun., vol. 33, no. 9, pp. 934-944, 1985.

[10] P. Gupta and P. R. Kumar. The capacity of wireless networks. IEEE Trans. Inf. Theory, vol. 46, no. 2, pp. 388-404, 2000.

[11] P. R. Kumar. A correction to the proof of a lemma in the capacity of wireless networks. IEEE Trans. Inf. Theory, vol. 49, no. 11, pp. 3117, 2003.

[12] M. Andrews and M. Dinitz. Maximizing capacity in arbitrary wireless networks in the SINR model: complexity and game theory. In Proc. of IEEE INFOCOM, pp. 1332-1340, 2009.

[13] J. Luo and A. Ephremides. On the throughput, capacity and stability regions of random multiple access. IEEE Trans. Inf. Theory, vol. 52, no. 6, pp. 2593-2607, 2006.

[14] Y. Shi, Y. T. Hou, J. Liu, and S. Kompella. How to correctly use the protocol interference model for multi-hop wireless networks. In Proc. of ACM MobiHoc, pp. 239-248, 2009.

[15] Y. Shi, Y. T. Hou, J. Liu, and S. Kompella. Bridging the gap between protocol and physical models for wireless networks. IEEE Trans. Mobile Comput., vol. 12, no. 7, pp. 1404-1416, 2013.

[16] A. Capone, I. Filippini, S. Gualandi, and D. Yuan. Resource optimization in multi-radio multi-channel wireless mesh networks. In: S. Basagni, M. Conti, S. Giordano, and I. Stojmenovic (editors), 
Mobile Ad Hoc Networking: The Cutting Directions, 2nd edition. Wiley and IEEE Press, pp. 241-274, 2013.

[17] I. Chlamtac and A. Lerner. A link allocation protocol for mobile multihop networks. In Proc. of IEEE GLOBECOM, pp. 238-242, 1985.

[18] D. Baker, A. Ephremides, and J. Flynn. The design and simulation of a mobile radio network with distributed control. IEEE J. Sel. Areas Commun., vol. 2, no. 1, pp. 226-237, 1984.

[19] I. Chlamtac and A. Lerner. Fair algorithms for maximal link activation in multihop radio networks. IEEE Trans. Commun., vol. 35, no. 7, pp. 739-746, 1987.

[20] A. M. Chou and V. O. K. Li. Fair spatial TDMA channel access protocols for multihop radio networks. In Proc. of IEEE INFOCOM, pp. 1064-1073, 1991.

[21] I. Cidon and M. Sidi. Distributed assignment algorithms for multihop packet radio networks. IEEE Trans. Comput., vol. 38, no. 10, pp. 1353-1361, 1989.

[22] I. Chlamtac, A. Farago, and H. Ahn. A topology transparent link activation protocol for mobile CDMA radio networks. IEEE J. Sel. Areas Commun., vol. 12, no. 8, pp. 1426-1432, 1994.

[23] B. Hajek. Balanced scheduling in a packet synchronized spread spectrum network. In Proc. of IEEE INFOCOM, pp. 56-65, 1983. 
[24] O. Goussevskaia, M. M. Halldórsson, R. Wattenhofer, and E. Welzl. Capacity of arbitrary wireless networks. In Proc. of IEEE INFOCOM, pp. 1872-1880, 2009.

[25] O. Goussevskaia, Y. A. Pswald, and R. Wattenhofer. Complexity in geometric SINR. In Proc. of ACM MobiHoc, pp. 100-109, 2007.

[26] G. Brar, D. Blough, and P. Santi. Computationally efficient scheduling with the physical interference model for throughput improvement in wireless mesh networks. In Proc. of ACM MobiHoc, pp. 2-13, 2006.

[27] X. Xu and S. Tang. A constant approximation algorithm for link scheduling in arbitrary networks under physical interference model. In Proc. of ACM FOWANC, pp. 13-20, 2009.

[28] T. Kesselheim. A constant-factor approximation for wireless capacity maximization with power control in the SINR model. In Proc. of ACM-SIAM SODA, pp. 1549-1559, 2011.

[29] A. Capone, L. Chen, S. Gualandi, and D. Yuan. A new computational approach for maximum link activation in wireless networks under the SINR model. IEEE Trans. Wireless Commun., vol. 10, no. 5, pp. 1368-1372, 2011.

[30] P. Björklund, P. Värbrand, and D. Yuan. Resource optimization of spatial TDMA in ad hoc radio networks: a column generation approach. In Proc. of IEEE INFOCOM, pp. 818-824, 2003.

[31] P. Värbrand and D. Yuan. Resource allocation of spatial time division multiple access in multi-hop radio networks. In: M. Cardei, I. 
Cardei, and D.-Z. Zhu (editors), Resource Management in Wireless Networking. Kluwer Academic Publishers, pp. 198-222, 2005.

[32] A. Pantelidou and A. Ephremides. The scheduling problem in wireless networks. Commun. Netw., vol. 11, no. 5, pp. 490-500, 2009.

[33] A. Capone, G. Carello, I. Filippini, S. Gualandi, and F. Malucelli. Routing, scheduling and channel assignment in wireless mesh networks: optimization models and algorithms. Ad Hoc Netw., vol. 8, no. 6, pp. 545-563, 2010.

[34] Y. Li and A. Ephremides. A joint scheduling, power control, and routing algorithm for ad hoc wireless networks. Ad Hoc Netw., vol. 5, no. 7, pp. 959-973, 2007.

[35] E. C. van der Meulen. Three-terminal communication channels. Adv. Appl. Probab., vol. 3, pp. 120-154, 1971.

[36] T. M. Cover and A. A. E. Gamal. Capacity theorems for the relay channel. IEEE Trans. Inf. Theory, vol. 25, no. 5, pp. 572-584, 1979.

[37] J. N. Laneman, D. N. C. Tse, and G. W. Wornell. Cooperative diversity in wireless networks: efficient protocols and outage behavior. IEEE Trans. Inf. Theory, vol. 50, no. 12, pp. 3062-3080, 2004.

[38] A. Sendonaris, E. Erkip, and B. Aazhang. User cooperation diversity, part i: system description/ part ii: implementation aspects 
and performance analysis. IEEE Trans. Comm., vol. 51, no. 11, pp. 1927-1948, 2003.

[39] G. Kramer, M. Gastpar, and P. Gupta. Cooperative strategies and capacity theorems for relay networks. IEEE Trans. Inf. Theory, vol. 51, no. 9, pp. 3037-3063, 2005.

[40] A. H. Madsen. Capacity bounds for cooperative diversity. IEEE Trans. Inf. Theory, vol. 52, no. 4, pp. 1522-1544, 2006.

[41] P. Herhold, E. Zimmermann, and G. Fettweis. Cooperative multihop transmission in wireless networks. Comput. Netw., vol. 49, no. 3, pp. 299-324, 2005.

[42] A. Capone, S. Gualandi, and Di Yuan. Joint routing and scheduling optimization in arbitrary ad hoc networks: comparison of cooperative and hop-by-hop forwarding. Ad Hoc Netw., vol. 9, no. 7, pp. 1256-1269, 2011.

[43] A. Nagate, S. Nabatame, D. Ogata, K. Hoshino, and T. Fujii. Field experiment of CoMP joint transmission over X2 interface for LTEAdvanced. In Proc. of IEEE VTC Spring, pp. 1-5, 2013.

[44] 3GPP, Coordinated multi-point operation for LTE physical layer aspects. Tech. Rep., TR 36.819, 2012.

[45] Qualcomm Incorporated. LTE Advanced: heterogeneous networks. Whitepaper, 2011.

[46] S. Verdu. Multiuser Detection. Cambridge University Press, 1998. 
[47] S. Sen, N. Santhapuri, R. R. Choudhury, and S. Nelakuditi. Successive interference cancellation: carving out MAC layer opportunities. IEEE Trans. Mobile Comput., vol. 12, no. 2, pp. 346-357, 2013.

[48] N. Pappas, M. Kountouris, and A. Ephremides. The stability region of the two-user interference channel. In Proc. of IEEE ITW, pp. 1-5, 2013.

[49] D. Yuan, V. Angelakis, L. Chen, E. Karipidis, and E. G. Larsson. On optimal link activation with interference cancellation in wireless networking. IEEE Trans. Veh. Technol., vol. 62, no. 2, pp. 939-945, 2013.

[50] E. Karipidis, D. Yuan, Q. He, and E. G. Larsson. Max-min power control in wireless networks with successive interference cancellation. IEEE Trans. Wireless Commun., vol. 14, no. 11, pp. 62696282, 2015.

[51] A. Pantelidou and A. Ephremides. Scheduling in wireless networks. Found. Trends. Network., vol. 4, no. 4, pp. 421-511, 2011.

[52] L. Georgiadis, M. J. Neely, and L. Tassiulas. Resource allocation and cross-layer control in wireless networks. Found. Trends. Network., vol. 1, no. 11, pp. 1-144, 2006.

[53] L. Tassiulas and A. Ephremides. Stability properties of constrained queueing systems and scheduling policies for maximum throughput in multihop radio. IIEEE Trans. Automat. Contr., vol. 37, no. 12, pp. 1936-1948, 1992. 
[54] T. Ho and H. Viswanathan. Dynamic algorithms for multicast with intra-session network coding. IEEE Trans. Inf. Theory, vol. 55, no. 2, pp. 797-815, 2009.

[55] M. J. Neely, E. Modiano, and C. E. Rohrs. Dynamic power allocation and routing for time-varying wireless networks. IEEE J. Select. Areas Commun., vol. 23, no. 1, pp. 89-103, 2005.

[56] B. Hajek and G. Sasaki. Link scheduling in polynomial time. IEEE Trans. Inf. Theory, vol. 34, no. 5, pp. 910-917, 1988.

[57] S. A. Borbash and A. Ephremides. Wireless link scheduling with power control and SINR constraints. IEEE Trans. Inf. Theory, vol. 52, no. 1, pp. 5106-5111, 2006.

[58] S. Kompella, J. E. Wieselthier, and A. Ephremides. A crosslayer approach to optimal wireless link scheduling with SINR constraints. In Proc. of IEEE MILCOM, pp. 1-7, 2007.

[59] S. Kompella, J. E. Wieselthier, and A. Ephremides. Revisiting the optimal scheduling problem. In Proc. of CISS, pp. 492-497, 2008.

[60] A. Pantelidou and A. Ephremides. Minimum-length scheduling and rate control for time-varying wireless networks. In Proc. of IEEE MILCOM, pp. 1-7, 2009.

[61] S. Toumpis and A. J. Goldsmith. Capacity regions for wireless ad hoc networks. IEEE Trans. Wireless Commun., vol. 2, no. 4, pp. 736-748, 2003. 
[62] G. D. Nguyen, S. Kompella, C. Kam, J. E. Wieselthier, and A. Ephremides. Minimum-energy link scheduling for emptying wireless networks. In Proc. of IEEE WIOPT, pp. 207-212, 2015.

[63] Y. Li, A. Capone, and D. Yuan. On end-to-end delay minimization in wireless network under physical interference model. In Proc. of IEEE INFOCOM, pp. 2020-2028, 2015.

[64] E. Arikan. Some complexity results about packet radio networks. IEEE Trans. Inf. Theory, vol. 30, no. 4, pp. 681-685, 1984.

[65] H. Hunt, M. Marathe, V. Radhakrishnan, S. Ravi, D. Rosenkrantz, and R. Stearns. NC-approximation schemes for NP- and PSPACEhard problems for geometric graphs. J. Algorithms, vo. 26, no. 2, pp. 238-274, 1998.

[66] S. O. Krumke, M. Marathe, and S. Ravi. Models and approximation algorithms for channel assignment in radio networks. Wireless Netw., vo. 7, no. 6, pp. 575-584, 2001.

[67] A. Sen and M. L. Huson. A new model for scheduling packet radio networks. In Proc. of IEEE INFOCOM, pp. 1116-1124, 1996.

[68] P. Björklund, P. Värbrand and D. Yuan. A column generation method for spatial TDMA scheduling in ad hoc networks. Ad Hoc Netw., vol. 2, no. 4, pp. 405-418, 2004.

[69] D. J. Baker and J. Wieselthier. A distributed algorithm for scheduling the activation of links in a self-organizing, mobile, radio networks. In Proc. of IEEE ICC, pp. 2F.6.1-2F.6.5, 1982. 
[70] M. Post, P. Sarachik and A. Kershenbaum. A biased greedy algorithm for scheduling multi-hop radio networks. In Proc. of IEEE CISS, pp. 564-572, 1985.

[71] I. Chlamtac and S. S. Pinter. Distributed nodes organization algorithm for channel access in a multihop dynamic radio network. IEEE Trans. Comput., vol. 36, no. 6, pp. 728-737, 1987.

[72] R. Liu and E. L. Lloyd. A distributed protocol for adaptive scheduling in ad hoc networks. In Proc. of IASTED WOC, pp. 43-48, 2001.

[73] G. Sharma, R. R. Mazumdar, and N. B. Shroff. On the complexity of scheduling in wireless networks. In Proc. of ACM MOBICOM, pp. 227-238, 2006.

[74] V. S. A. Kumar, M. V. Marathe, S. Parthasarathy, and A. Srinivasan. End-to-end packet-scheduling in wireless ad-hoc networks. In Proc. of ACM-SIAM SODA, pp. 1021-1030, 2004.

[75] C. G. Prohazka. Decoupling link scheduling constraints in multihop packet radio networks. IEEE Trans. Comput., vol. 38, no. 3, pp. 455-458, 1989.

[76] D. S. Stevens and M. H. Ammar. Evaluation of slot allocation strategies for TDMA protocols in packet radio networks. In Proc. of IEEE MILCOM, pp. 835-839, 1990.

[77] S. Ramanathan and E. L. Lloyd. Scheduling algorithms for multihop radio networks. IEEE/ACM Trans. Netw., vo. 1, no. 2, pp. 166-177, 1993. 
[78] A. Ephremides and T. Truong. Distributed algorithm for efficient and interference-free broadcasting in radio networks. In Proc. of IEEE MILCOM, pp. 1119-1124, 1988.

[79] R. Ramaswami and K.K. Parhi. Distributed scheduling for broadcasts in a radio network. In Proc. of IEEE MILCOM, pp. 497-504, 1989.

[80] A. Ephremides and T. V. Truong. Scheduling broadcasts in multihop radio networks. IEEE Trans. Commun., vol. 38, no. 4, pp. 456-460, 1990.

[81] N. Funabiki and Y. Takefuji. A parallel algorithm for broadcast scheduling problems in packet radio networks. IEEE Trans. Commun., vol. 41, no. 6, pp. 828-831, 1993.

[82] D. Yang, X. Fang, N. Li, and G. Xue. A simple greedy algorithm for link scheduling with the physical interference model. In Proc. of IEEE GLOBECOM, pp. 4572-4577, 2009.

[83] P. J. Wan, P. Wan, O. Frieder, X. Jia, F. Yao, X. Xu, and S. Tang. Wireless link scheduling under physical interference model. In Proc. of IEEE INFOCOM, pp. 838-845, 2011.

[84] T. Moscibroda, R. Wattenhofer, and A. Zollinger. Topology control meets sinr: the scheduling complexity of arbitrary topologies. In Proc. of ACM MobiHoc, pp. 310-321, 2006.

[85] C. Boyaci, B. Li, and Y. Xia. An investigation on the nature of wireless scheduling. In Proc. of IEEE INFOCOM, pp. 1-9, 2010. 
[86] A. Pantelidou and A. Ephremides. Minimum schedule lengths with rate control in wireless networks. In Proc. of IEEE MILCOM, pp. 1-7, 2008.

[87] S. Kompella, J. E. Wieselthier, A. Ephremides, H. D. Sherali, and G. D. Nguyen. On optimal SINR-based scheduling in multihop wireless networks. IEEE/ACM Trans. Netw., vol. 18, no. 6, pp. 1713-1724, 2010.

[88] Y. Li, M. Pióro, D. Yuan, and J. Su. Optimizing compatible sets in wireless networks through integer programming. European $J$. Comput. Optim., vol 2, no. 1-2, pp. 1-15, 2013.

[89] T. Elbatt and A. Ephremides. Joint scheduling and power control for wireless ad-hoc networks. In Proc. of IEEE INFOCOM, pp. 976-984, 2002.

[90] A. Capone and G. Carello. Scheduling optimization in wireless mesh networks with power control and rate adaptation. In Proc. of IEEE MASS, pp. 138-147, 2006.

[91] X. Lin and N. B. Shroff. Joint rate control and scheduling in multihop wireless networks. In Proc. of IEEE CDC, pp. 14841489, 2004.

[92] U. C. Kozat and I. Koutsopoulos. Cross-layer design for power efficiency and QoS provisioning in multi-hop wireless networks. IEEE Trans. Wireless Commun., vol. 5, no. 11, pp. 3306-3315, 2006. 
[93] K. K. Leung and L. Wang. Integrated link adaptation and power control for wireless IP networks. In Proc. of IEEE VTC Spring, pp. 1090-3038, 2000.

[94] A. Capone, I. Filippini, and F. Martignon. Joint routing and scheduling optimization in wireless mesh networks with directional antennas. In Proc. of IEEE ICC, pp. 2951-2957, 2008.

[95] D. Yuan, V. Angelakis, and L. Lei. Minimum-length scheduling in wireless networks with multi-user detection and interference cancellation: optimization and performance evaluation. In Proc. of IEEE ICCS, pp. 315-319, 2012.

[96] S. Lv, W. Zhuang, X. Wang, and X. Zhou. Scheduling in wireless ad hoc networks with successive interference cancellation. In Proc. of IEEE INFOCOM, pp. 1287-1295, 2011.

[97] B. Gopalakrishnan and N. D. Sidiropoulos. Joint back-pressure power control and interference cancellation in wireless multi-hop networks. IEEE Trans. Wireless Commun., vol. 12, no. 7, pp. 34843495, 2013.

[98] V. Angelakis, L. Chen, and D. Yuan. Optimal and collaborative rate selection for interference cancellation in wireless networks. IEEE Commun. Lett., vol. 15, no. 8, pp. 819-821, 2011.

[99] Y. T. Hou, Y. Shi, and H. D. Sherali. Applied Optimization Methods for Wireless Networks. Cambridge University Press, 2014. 
[100] M. Pióro and D. Medhi. Routing, Flow, and Capacity Design in Communication and Computer Networks. Morgan Kaufmann, 2004.

[101] D. Bertsimas and J. N. Tsitsiklis. Introduction to Linear Optimization. Athena Scientific Press, 1997.

[102] L. A. Wolsey. Integer Programming. Wiley Press, 1998.

[103] S. Boyd and L. Vandenberghe. Convex Optimization. Cambridge University Press, 2004.

[104] IBM, IBM CPLEX Optimizer 12.6, http://www01.ibm.com/software/commerceloptimization/cplex-optimizer/, 2015.

[105] GUROBI, Gurobi Optimizer 6.5, http://www.gurobi.com/, 2015.

[106] R. K. Ahuja, T. L. Magnanti, and J. B. Orlin. Network Flows: Theory, Algorithms, and Applications. Pearson, 2014.

[107] P. M. Pardalos and M. G. C. Resende. Handbook of Applied Optimization. Oxford University Press, 2002.

[108] M. R. Garey and D. S. Johnson. Computers and Intractability: A Guide to the Theory of NP-completeness. Macmillan Higher Education, 1979. 


\section{Part II}

\section{Research Papers}





\section{Research Papers}

The articles associated with this thesis have been removed for copyright reasons. For more details about these see:

http://urn.kb.se/resolve?urn=urn:nbn:se:liu:diva-131359 